\title{
Molecular Defects Associated with Antithrombin Deficiency and Dilated Cardiomyopathy in a Japanese Patient
}

\author{
Yuta Fujimori ${ }^{1}$, Hidemi Okimatsu ${ }^{2}$, Takahiro Kashiwagi ${ }^{1}$, Naomi Sanda ${ }^{1}$, Kaoru Okumura ${ }^{1}$, \\ Akira Takagi $^{1,2}$, Kohzo Nagata ${ }^{1,2}$, Takashi Murate ${ }^{1,2}$, Aya Uchida ${ }^{3}$, Koichi Node ${ }^{3}$, \\ Hidehiko Saito ${ }^{4}$ and Tetsuhito Kojima ${ }^{1,2}$
}

\begin{abstract}
Objective The molecular basis for the antithrombin (AT) deficiency and dilated cardiomyopathy (DCM) combined in a Japanese patient was investigated.

Methods We analyzed candidate genes-SERPINC1 for AT deficiency, and TNNT2 and LMNA for DCM. In addition, we examined the characteristics of recombinant mutant AT and evaluated the LMNA mutation associated with DCM by molecular modeling.

Results Genome sequencing of SERPINC1 revealed a C-to-A transversion in exon 6 that resulted in a p.Pro439Thr mutation of AT, which was previously reported as a pleiotropic effect type II AT deficiency (AT Budapest5). However, expression experiments with recombinant 439Thr-AT showed normal heparin affinity, slightly reduced secretion, and low specific activity, which suggested that this mutation exhibits an intermediate feature of type I and type II AT deficiencies. In a survey of gene abnormalities causing DCM, we found no causative gene defect in TNNT2; however, we identified a G-to-C transversion in LMNA that resulted in a novel p.Asp357His mutation in lamin A/C. This acidic-to-basic residue substitution might have impaired the head-to-tail association of two lamin dimers leading to DCM. Further, we identified both SERPINCI and LMNA mutations in the patient's daughter and son, both of whom had AT deficiency. These data suggested that a p.Pro439Thr mutation in SERPINC1 and a p.Asp357His mutation in LMNA might have cosegregated in this family, associated with AT deficiency and DCM, respectively.

Conclusions We identified missense mutations in SERPINC1 and LMNA genes to be associated with AT deficiency and DCM, respectively, which might have cosegregated in the family of the patient.
\end{abstract}

Key words: antithrombin (AT), pleiotropic effect, dilated cardiomyopathy (DCM), lamin A/C

(Inter Med 47: 925-931, 2008)

(DOI: 10.2169/internalmedicine.47.0669)

\section{Introduction}

Antithrombin (AT) is a plasma serine protease inhibitor that inhibits thrombin as well as other activated serine proteases of the coagulation system (1). Plasma AT plays a key role in the natural hemostatic balance to maintain blood fluidity. Therefore, patients with AT deficiency are susceptible to thromboembolic diseases, particularly deep vein thrombo- sis of the lower limb and pulmonary embolism. The human AT gene (SERPINC1) is located on chromosome 1q23-25 (2), and many defects of this gene have been reported in patients with AT deficiency (3). Congenital AT deficiency is usually heterozygous and classified into two types: quantitative deficiency (type I) and qualitative deficiency (type II). The latter includes reactive site defect, heparin binding site defect, and pleiotropic effect AT deficiencies.

Dilated cardiomyopathy (DCM), the most frequent form

\footnotetext{
${ }^{1}$ Department of Pathophysiological Laboratory Sciences, Nagoya University Graduate School of Medicine, Nagoya, ${ }^{2}$ Department of Medical Technology, Nagoya University School of Health Sciences, Nagoya, ${ }^{3}$ Department of Cardiovascular and Renal Medicine, Saga University Faculty of Medicine, Saga and ${ }^{4}$ Nagoya Central Hospital, Nagoya

Received for publication October 13, 2007; Accepted for publication February 18, 2008

Correspondence to Dr. Tetsuhito Kojima, kojima@met.nagoya-u.ac.jp
} 
Table 1. Clinical Data of the Family Members Analyzed

\begin{tabular}{|c|c|c|c|c|c|}
\hline \multirow{2}{*}{ Member } & \multirow{2}{*}{$\begin{array}{l}\text { Age } \\
\text { (yrs) }\end{array}$} & \multicolumn{2}{|c|}{ Antithrombin } & \multirow{2}{*}{ Thrombosis } & \multirow{2}{*}{$\begin{array}{l}{ }^{*} \mathrm{EF} \\
(\%)\end{array}$} \\
\hline & & antigen (\%) & activity (\%) & & \\
\hline III2 & 47 & 49.7 & 52.0 & ${ }^{* *} \mathrm{BI}, 44 \mathrm{yrs}$ & 38 \\
\hline IV1 & 27 & 58.6 & 43.9 & None & 65 \\
\hline IV3 & 22 & 59.0 & 59.8 & None & ${ }^{* * *} N D$ \\
\hline
\end{tabular}

*EF: ejection fraction (M.Simpson), ${ }^{* *} \mathrm{BI}$ : brain infarction, ${ }^{* *} \mathrm{ND}$ : not done

of cardiomyopathy, is a myocardial disorder characterized by ventricular dilatation and impaired systolic function, which leads to congestive heart failure and sudden death (4). Familial cases of DCM were initially considered as quite rare; however, recent studies with systematic and careful screening of relatives of the patients have shown that up to $35 \%$ of patients with DCM have a familial disease (5). Familial DCM has been reported most commonly with autosomal dominant inheritance, and mutations in 16 autosomal genes have been the proximate cause until date (6). Among them, the most frequently reported genetic causes of DCM are mutations in the gene encoding lamin $\mathrm{A} / \mathrm{C}$ proteins. The lamin $\mathrm{A} / \mathrm{C}$ gene (LMNA), that is encoded on chromosome 1q21.2-q21.3 (7), has been reported to be involved in DCM associated with conduction system disease (8), and LMNA mutations can be found in up to $33 \%$ of cases of DCM in association with cardiac conduction disease (9).

AT deficiency is a risk of venous thromboembolism, and DCM is also a risk of systemic or pulmonary embolization, because blood stasis and low shear rate in the hypocontractile ventricle lead to the activation of coagulation processes (4). Therefore, the patient with both diseases in combination is likely to be in a highly thrombophilic state. Congenital AT deficiency and DCM are rare diseases inherited independently each other, and have never been reported in combination to date.

Here, we report a Japanese patient with combined congenital AT deficiency and DCM. We investigated the molecular defects associated with these two diseases, and identified the distinct missense mutations in SERPINC1 and LMNA genes that might be responsible for AT deficiency and DCM, respectively.

\section{Materials and Methods}

\section{Patient and sample preparations}

The patient was a 47-year-old man, who had a history of brain infarction associated with cardiac arrhythmia at the age of 44, and was treated with an oral anticoagulant. He showed cardiomegaly on the chest radiography and left ventricular dilatation as well as hypokinesis $(\mathrm{EF}=38 \%)$ on the echocardiography, and was diagnosed as having DCM. A transesophageal echocardiography revealed a smoke-like echo that appeared to be a thrombus, although he had been taking warfarin. As the result of more intense warfarization, the thrombus disappeared. Finally, he was implanted a cardioverter defibrillator (ICD) to treat the sustained ventricular tachycardia. He was also diagnosed as having AT deficiency by blood coagulation tests (Table 1). In the family history, his monozygotic twin brother and maternal cousin had been also diagnosed as having AT deficiency and implanted a pacemaker because of complete AV-block (Fig. 1).

Ethical approval for the study was obtained from the Ethics Committee of the Nagoya University School of Medicine, as well as from the Ethics Committee of the Saga University Faculty of Medicine. Blood samples were obtained with an informed consent from the patient in accordance with the Declaration of Helsinki. Genomic DNA was isolated from peripheral blood leukocytes (10). We obtained DNA samples only from the proband and his two children, who were also diagnosed as having AT deficiency (Table 1).

\section{Identification of gene abnormalities in the patient}

All exons including splice junctions of the SERPINC1 gene were amplified by polymerase chain reaction (PCR) and analyzed by direct sequencing (11). Similarly, TNNT2 and $L M N A$ genes were also analyzed by direct sequencing, after PCR amplification of all exons including splice junctions using the primer sets listed in Table 2.

\section{Transient expression of recombinant ATs}

We prepared a mutant human AT expression vector (pcDNA/439Thr-AT: the initial Met residue is denoted amino acid +1) using the recombinant PCR method (11). For heparin affinity experiments, we used the wild type AT (pcDNA/WT-AT) and the heparin affinity deficient AT Nagasaki (pcDNA/148Pro-AT) vectors as positive and negative controls, respectively (11).

Human embryo kidney 293 (HEK293) cells were grown in Dulbecco's modified Eagle medium (DMEM) supplemented with $10 \%$ fetal calf serum (FCS) and $5 \% \mathrm{CO}_{2}$, at 37 ${ }^{\circ} \mathrm{C}$. The cells were cultured in $60-\mathrm{mm}$ dishes until they became $50 \%$ confluent and then transiently transformed with $10 \mu \mathrm{g}$ of the expression plasmid vectors by the calcium phosphate method (12). After a 24 hours incubation in FCSfree DMEM, the cell culture media and cell lysates that had dissolved in the Reporter lysis buffer (Promega; Madison, WI, USA) were collected, centrifuged at $1500 \times \mathrm{g}$ for 10 minutes, and used as Western blot samples. Western blot analysis was performed (13), using a polyclonal anti-AT antibody (rabbit $\mathrm{IgG}$ ) and a peroxidase-labeled anti-rabbit $\mathrm{IgG}$ 


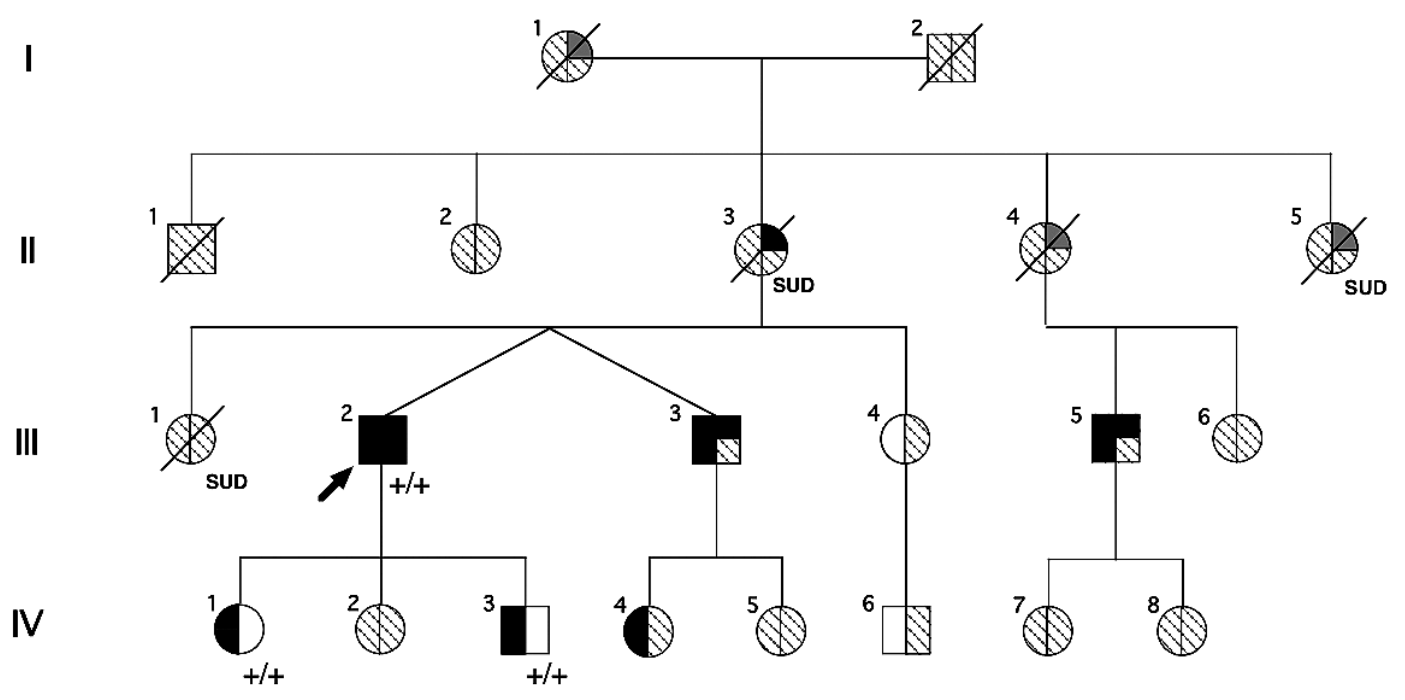

Figure 1. Pedigree of the family. Squares and circles show males and females, respectively. The proband is indicated by an arrow. Left half-black symbols, AT deficiency members; right upper quarter-black symbols, cardiac conduction disease members implanted with pace maker or ICD (proband); right upper quarter-gray symbols, unspecified heart disease members; right lower quarter-black symbol, DCM member; white symbols, unaffected members; shaded symbols, members of unknown clinical status; diagonal lines, dead members; SUD, sudden death, +/+, presence of SERPINC1 p.Pro439Thr and LMNA p.Asp357His heterozygous mutations. Genotypes of 3 members were tested, but no sample from other members was available.

Table 2. Oligonucleotide Primers of PCR Amplifying TNNT2 and LMNA Genes

\begin{tabular}{|c|c|c|c|c|c|}
\hline Gene & Exon & Sense $\left(5^{\prime} \rightarrow 3^{\prime}\right)$ & Antisense $\left(3^{\prime} \rightarrow 5^{\prime}\right)$ & Product (bp) & Annealing $\left({ }^{\circ} \mathrm{C}\right)$ \\
\hline \multirow[t]{11}{*}{ TNNT2 } & 2 & TTTTGTTGCAGGTCACACAG & AGGGGTACAGGAGTGGAAAG & 367 & 56 \\
\hline & $3+4$ & GCAACAAGGGAAAAGAAAGG & GAAGGCACTGTTGTTGGAGG & 391 & 56 \\
\hline & 5 & AATGCCGGCCTAACTCCAA & GGAGGCAGGGGAGGAAAC & 308 & 59 \\
\hline & $6+7$ & TGCTCTGGGTTCTGCCTG & CTGCTGTGAGGGGTTCCTT & 578 & 56 \\
\hline & 8 & GTGCAGATGGGGAAATGGA & CCTTAGGAAGAGACGCTTGTG & 313 & 56 \\
\hline & $9+10$ & TCAGTCCCTGGGTCCAGAA & GGATGGAGGACAGACTGGG & 590 & 59 \\
\hline & 11 & AAAGTGGAGGCCCTTGGA & GATGAATAGAGAGGGGCCTG & 315 & 59 \\
\hline & 12 & CAAGCTTCAGCCCAGAATCA & CAGTCTTCCACCCACAGCA & 314 & 56 \\
\hline & 13 & TTGGTCTTTTTCTATGGGCCT & AGAGCAGATGCGGGCAGT & 574 & 56 \\
\hline & 14 & TTGGCAGGCCTGGAGGT & GGCAGATGCAGGAGCTGA & 347 & 59 \\
\hline & $15+16$ & GCCAGTCAGCTCCAGCGT & GCGAGGAGCAGATCTTTGGT & 537 & 59 \\
\hline \multirow[t]{12}{*}{ LMNA } & 1 & GCACTCCGACTCCGAGCA & CGCCGCССTCTCСАСТC & 502 & 64 \\
\hline & 2 & GACCTCCTGGGAGCCTG & GGAGGGCCTAGGTAGAAGAGT & 306 & 61 \\
\hline & 3 & GCAGCAGCCCACCTCTC & AAGGCGAGCTCTGCACAC & 301 & 61 \\
\hline & 4 1st & GACAGGGAGTTGGGGGTGG & TGACTGGGAGGGGTGGAGG & 510 & 63 \\
\hline & 2nd & GAGTAGGGCTGGGCAGG & AGCGTGGGTAAGGGTAGG & 330 & 60 \\
\hline & 5 & CCTGGGGCTGTAGCAGTGA & CTGTGGTTGTGGGGACACTTT & 354 & 60 \\
\hline & 6 & CTACACCGACCCACGTCC & CCAAGTGGGGGTCTAGTCAA & 374 & 59 \\
\hline & 7 & GGGAGGTGCTGGCAGTGT & СТСТСТССАСАТGССАТССТT & 378 & 59 \\
\hline & $8+9$ & TGAGCCTCCCCGACCTT & TCCGATGTTGGCCATCAG & 413 & 59 \\
\hline & 10 & AAAGGGCAGGCCACAAGA & CAGAGTAGGGCACCCAGACA & 394 & 64 \\
\hline & 11 & TTGGGCCTGAGTGGTCAGT & CTCGTCCTACCCCTCGATG & 398 & 59 \\
\hline & 12 & GAGGGGTAGGACGAGGTGG & TGAGGTGAGGAGGACGCAG & 354 & 64 \\
\hline
\end{tabular}

antibody (Behringwerke AG; Marburg, Germany).

\section{Specific anticoagulant activities and heparin affini- ties of recombinant ATs}

We established stable transformants of HEK293 cells highly expressing the recombinant AT molecules and measured their antigen levels in the culture media by enzyme- linked immunosorbent assay (11). Heparin cofactor activity of the AT was measured using Ntest AT III-S kit (Nittobo; Tokyo, Japan). Progress activity of the AT was measured in the absence of heparin. The specific activity of each recombinant AT molecule was calculated as a percentage of the wild type activity.

We also performed affinity chromatography of each re- 


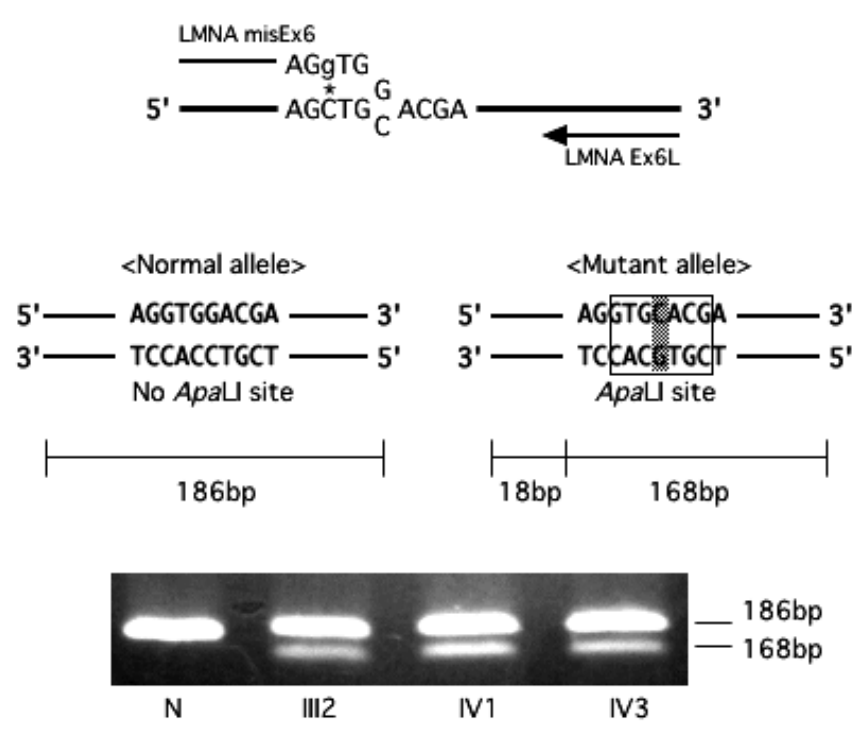

Figure 2. Mismatch PCR-ApaLI RFLP detected LMNA mutation. ApaLI-RFLP (PCR-mediated) using partially mismatched sense primer (LMNA misEx6) to detect a c.1,069G $>$ C mutation in exon 6. PCR products from mutant allele DNA will yield a 168-bp fragment after being digested by ApaLI, whereas those from normal alleles yield an uncleaved 186-bp size band. Lane $\mathrm{N}$ shows a normal subject.

combinant AT molecule on a heparin-sepharose column as described previously (11). The bound AT was eluted by a stepwise increasing concentration of $\mathrm{NaCl}(0.25,0.5,0.75$, 1.0 , and $1.5 \mathrm{~mol} / \mathrm{l})$. The concentration of AT in each fraction was determined by dot blot assay, which showed data similar to ELISA.

\section{Mismatch PCR-ApaLI-Restriction Fragment Length Polymorphism}

We analyzed the identified mutation in LMNA gene by PCR-mediated restriction fragment length polymorphism (RFLP) as described previously (14). Thus, we designed a mismatch PCR strategy to introduce a new restriction enzyme (ApaLI) site into the PCR products through the mutant allele (Fig. 2). We used a partially mismatched sense primer (LMNA mis6U: 5'-CAAGGATGCAGCAGCAGgTG), which introduced an ApaLI site only into the mutant allele PCR products, to amplify a part of exon 6 fragment of LMNA gene. Subsequently, the PCR products were digested with ApaLI, and resolved by $3 \%$ agarose gel electrophoresis with ethidium bromide.

\section{Results}

We analyzed SERPINC1 gene in the proband by PCRmediated direct sequencing, and identified a C-to-A transversion in exon 6 [c.1315C $>\mathrm{A}$; according to recommendations for the description of DNA sequence variants by $\mathrm{Hu}$ man Genome Variation Society (15)] in the heterozygous state, which was previously known as AT Budapest 5 (p.Pro439Thr) mutation with a pleiotropic effect phenotype
(16). We also detected the same mutation in both his daughter and son in the heterozygous state, which was confirmed by StuI RFLP analysis (data not shown).

In this study, we investigated the influences of p.Pro439Thr mutation on secretion and function of the recombinant AT molecule. We observed a decrease in the secretion of the mutant 439Thr-AT (74\%), and a slight decrease in progressive activity (84\%) as well as heparin cofactor activity $(83 \%)$ of the mutant, as compared with the wild type AT (Fig. 3). We also compared the heparin affinity of the recombinant ATs using heparin-sepharose affinity chromatography (Fig. 3C). We observed an unexpected normal heparin affinity in the recombinant 439Thr-AT, whereas the recombinant 148Pro-AT (AT Nagasaki) showed an impaired affinity to heparin as reported previously $(11,17)$.

Subsequently, we attempted to analyze two candidate genes causing DCM (TNNT2 and LMNA), which are located on the same chromosome as SERPINC1 (chromosome 1). In $T N N T 2$, a 5 bp deletion polymorphism in intron 3, which was reported as a risk of left ventricular hypertrophy in the homozygous state (18), was detected in the heterozygous state, although no other sequence alteration was found (data not shown). In LMNA gene, a G-to-C transversion in exon 6 (c.1069 G>C), resulting in a novel p.Asp357His mutation of the lamin $\mathrm{A} / \mathrm{C}$ molecule, was identified in the heterozygous state. We also detected a heterozygous missense mutation in both his daughter and son, which was confirmed by the mismatch PCR-ApaLI-RFLP (Fig. 2). Additionally, the PCRApaLI-RFLP assay of 72 samples from healthy volunteers showed a single band (186 bp), suggesting that the c.1069 G $>\mathrm{C}$ mutation would not be a single nucleotide polymorphism (data not shown).

\section{Discussion}

We investigated the molecular basis of the AT deficiency and DCM in a family with both the diseases and identified p.Pro439Thr missense mutation in SERPINC1 gene and p. Asp357His missense mutation in LMNA gene.

The p.Pro439Thr mutation in SERPINC1 gene has been reported as AT Budapest 5 with a pleiotropic effect, which altered the reactive site and heparin-binding properties of the variant (16). It was reported that the patient with AT Budapest 5 had a normal level of AT antigen and a slightly decreased AT activity in the plasma; however, the proband in this study showed half levels of both plasma AT activity and antigen. The reason for this discrepancy is not clear, but it might be possible that some different environmental circumstances or other genetic factors influenced circulating levels of ATs. Some type II AT mutations were reported as having very low levels of AT antigen like a type I AT mutation, even though they had an identical mutation (3). In addition, the secretion of recombinant 439Thr-AT in our expression experiments was moderately reduced, whereas its heparin affinity was normal, and its progressive activity and heparin cofactor activity were mildly impaired. These data suggest 
A

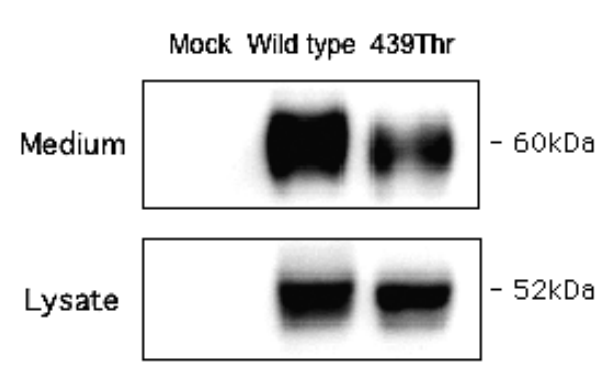

B

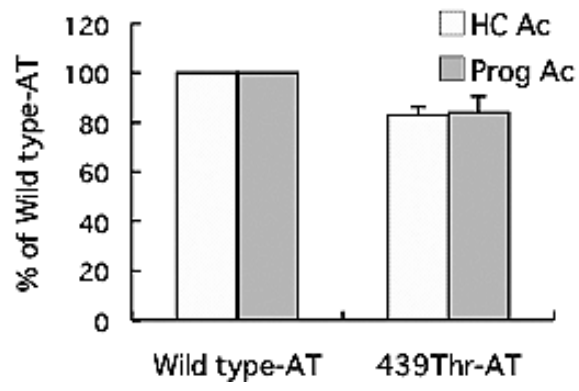

C

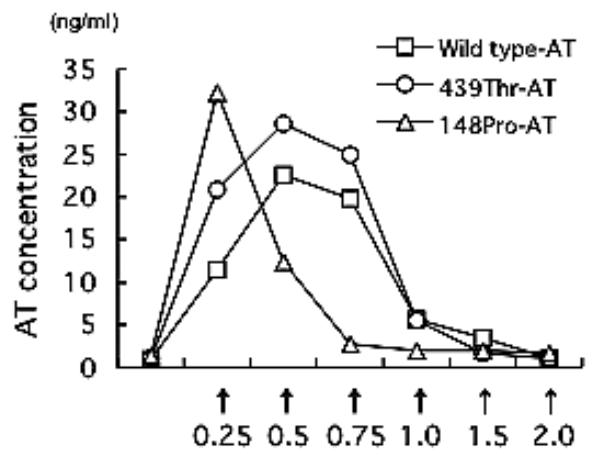

$\mathrm{NaCl}$ concentration $(\mathrm{M})$

Figure 3. Molecular characterization of recombinant ATs. (A) Western blot analysis of the recombinant AT molecules. Wild type-AT and 439Thr-AT were transiently expressed in HEK293T cells, and the culture media and lysates were analyzed. Ten micrograms of each sample was loaded to normalize total protein. (B) Progressive AT activities (Prog Ac) and heparin cofactor AT activities (HC Ac) of the recombinant proteins stably expressed in the culture media were examined as described in Materials and Methods. Specific AT activities were calculated as a percentage of wild type-AT activity (mean \pm SD \%, n=3). (C) Affinity chromatography of the recombinant AT molecules was performed on a heparin-sepharose column as described in Materials and Methods.

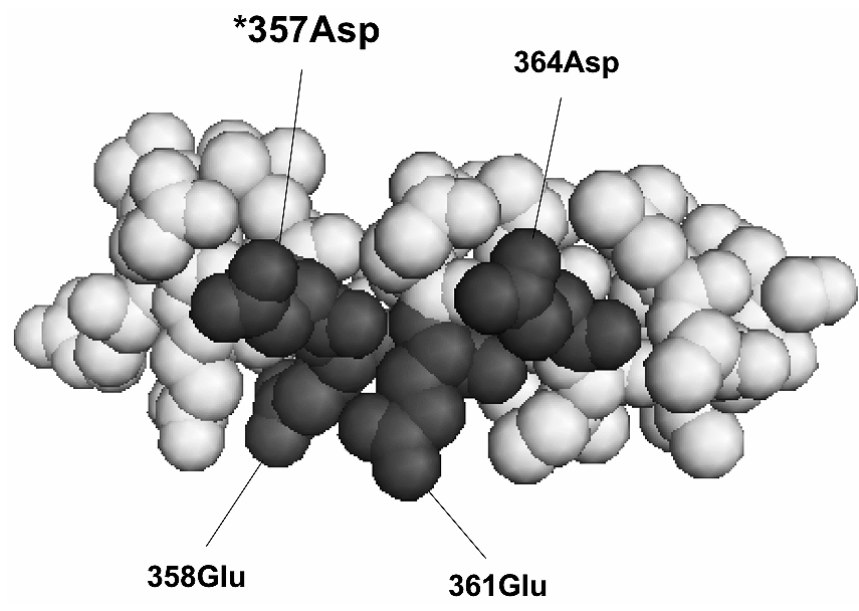

Figure 4. Molecular model of human lamin A/C coil 2B domain. Three-dimensional model of the partial peptide of the lamin A/C coil 2B domain (residues 351 to 370; protein data bank ID, $1 \times 8 y$ ). The amino acids were shown as the spheres, and the acidic residues are colored in gray and others in white. The figure was designed by using MacPyMoL 0.99 (DeLano Scientific LLC, Palo Alto, CA, USA; http://pymol.sourceforge.net/).

that the p.Pro439Thr mutation would cause intermediate features of type I and type II AT deficiencies in the patients.

We subsequently tried to determine the cause of DCM in the same patient. We analyzed two candidate genes causing DCM (TNNT2 and LMNA), located on the same chromosome as SERPINC1 (chromosome 1), and identified a novel p.Asp357His mutation in LMNA gene, although no causative abnormality in the TNNT2 was detected. There is no direct evidence that this LMNA mutation causes DCM, however, some relatives including his monozygotic twin brother suffered from cardiac conduction disturbance, which is common in DCM caused by LMNA mutations, suggesting that the LMNA p.Asp357His mutation could be associated with DCM.

Amino acid alignments of a part of the lamin A/C coil 2B fragment around an Asp357 in several species, including human, monkey, dog, mouse, chicken, frog and fish, shows that amino acid sequences are highly conserved among all species. In crystallographic modeling approach, the Asp357 lies within the highly conserved and exclusive acidic Cterminal sequences of the rod domain, which form one pronounced patch of negative electrostatic potential (Fig. 4). It was hypothesized that this acidic patch may be electrostatically attracted by a net positively charged cluster of Arg residues of the $N$-terminal head domains, and this interaction is important in the linear assembly of lamins (19). Therefore, we assumed that the substitution of an acidic aspartate with a basic histidine residue would impair normal lamina formation; hence, p.Asp357His mutation in LMNA gene 
could be associated with DCM in the patient. It has also been reported that Emery-Dreifuss muscular dystrophy, one of the laminopathies, is caused by LMNA p.Glu358Lys mutation (20).

The proband had combined AT deficiency and DCM, and the monozygotic twin brother as well as the maternal cousin also suffered from AT deficiency and cardiac conduction disturbance, which is commonly seen in DCM due to LMNA mutations. These findings suggested that the AT deficiency co-segregated with the LMNA p.Asp357His mutation, which could be associated with DCM, in this family.

Congenital AT deficiency by the SERPINC1 (1q23-25) mutation and DCM by the LMNA (1q21.2-21.3) mutation are rare diseases by independent inheritance, although both genes are located in the nearby regions on the same Chromosome 1. This is the first report of a compound case of two gene mutations associated with AT deficiency and DCM. Since both AT deficiency and DCM are risks of thromboembolization $(3,4)$, patients with both diseases in combination could be highly thrombophilic. As expected, the proband showed the thrombus formation in the left auricular appendix on the transesophageal echocardiography, although he had been taking warfarin.

Interstitial myocardial fibrosis is an important pathologic finding in DCM, although it is not specific, and problems with its quantification and prognostic value remains unresolved (21). In terms of myocardial fibrosis and AT deficiency, it is noteworthy that myocardial fibrosis in low tissue factor mice improved after plasma levels of AT were decreased in the mouse model, although the mechanism was not clear (22). Also, histological examination revealed only focal fibrosis in the myocardial biopsy samples of the pro- band (data not shown). The genetic and clinical heterogeneity of familial DCM suggests that it might be caused by a single gene, with multiple other genetic and environmental factors altering its expressivity (23). Taken together, it might also be possible that AT deficiency would show some effects against interstitial fibrosis of the heart in DCM patients.

In conclusion, we investigated the molecular basis of AT deficiency and DCM in a patient with both diseases, and identified missense mutations in the SERPINC1 and LMNA genes, which may cosegregate in the family. It remains to be possible that DCM in the proband is caused by another molecule mutation, but it is more likely that the LMNA gene mutation is associated with DCM. It is because his monozygotic twin brother, who should have the same LMNA mutation, showed cardiac conduction disturbance, which is common in DCM due to LMNA mutations. To prove that the LMNA p.Asp357His mutation can cause DCM, and that the SERPINC1 p.Pro439Thr mutation can affect the pathophysiology of DCM, knock-in experiments of the mouse model will be necessary in future.

\section{Acknowledgement}

We would like to thank C. Wakamatsu for her expert technical assistance and are indebted to R. Tanaka, D. Nakashima, and T. Yamada for the informative discussions.

This study was supported in part by Grants-in-Aid from the Japanese Ministry of Education, Culture, Sports, Science and Technology (19590553), and the Japanese Ministry of Health, Labor and Welfare (Research on Measures for Intractable Diseases).

\section{References}

1. Rosenberg RD. Biochemistry of heparin antithrombin interactions, and the physiologic role of this natural anticoagulant mechanism. Am J Med 87: S2-S9, 1989.

2. Bock S, Harris J, Balazs I, Trent J. Assignment of the human antithrombin III structural gene to chromosome 1q23-25. Cytogenet Cell Genet 39: 67-69, 1985.

3. Lane D, Bayston T, Olds R, et al. Antithrombin mutation database: 2nd (1997) update. For the Plasma Coagulation Inhibitors Subcommittee of the Scientific and Standardisation Committee of the International Society on Thrombosis and Haemostasis. Thromb Haemost 77: 197-211, 1997.

4. Dec GW, Fuster V. Idiopathic dilated cardiomyopathy. N Engl J Med 331: 1564-1575, 1994.

5. Richard P, Villard E, Charron P, Isnard R. The genetic bases of cardiomyopathies. J Am Coll Cardiol 48: A79-89, 2006.

6. Burkett EL, Hershberger RE. Clinical and genetic issues in familial dilated cardiomyopathy. J Am Coll Cardiol 45: 969-981, 2005.

7. Wydner KL, McNeil JA, Lin F, Worman HJ, Lawrence JB. Chromosomal assignment of human nuclear envelope protein genes LMNA, LMNB1, and LBR by fluorescence in situ hybridization. Genomics 32: 474-478, 1996.

8. Fatkin D, MacRae C, Sasaki T, et al. Missense mutations in the rod domain of the lamin $\mathrm{A} / \mathrm{C}$ gene as causes of dilated cardiomyopathy and conduction-system disease. $\mathrm{N}$ Engl J Med 341: $1715-1724,1999$.
9. Arbustini E, Pilotto A, Repetto A, et al. Autosomal dominant dilated cardiomyopathy with atrioventricular block: a lamin $\mathrm{A} / \mathrm{C}$ defect-related disease. J Am Coll Cardiol 39: 981-990, 2002.

10. Kojima T, Tanimoto M, Kamiya T, et al. Possible absence of common polymorphisms in coagulation factor IX gene in Japanese subjects. Blood 69: 349-352, 1987.

11. Kyotani M, Okumura K, Takagi A, et al. Molecular basis of antithrombin deficiency in four Japanese patients with antithrombin gene abnormalities including two novel mutations. Am J Hematol 82: 702-705, 2007.

12. Sobue S, Hagiwara K, Banno Y, et al. Transcription factor specificity protein 1 ( $\mathrm{Sp} 1)$ is the main regulator of nerve growth factorinduced sphingosine kinase 1 gene expression of the rat pheochromocytoma cell line, PC12. J Neurochem 95: 940-949, 2005.

13. Yamada $T$, Takagi A, Takeshita $K$, et al. Enzyme immunoassay for measurement of murine plasminogen activator inhibitor-1, employing a specific antibody produced by the DNA vaccine method. Thromb Res 111: 285-291, 2003.

14. Okada $\mathrm{H}$, Takagi A, Murate $\mathrm{T}$, et al. Identification of protein $\mathrm{SCE} \pm$ gene mutations including four novel mutations in eight unrelated patients with protein S deficiency. Br J Haematol 126: 219-225, 2004.

15. den Dunnen J, Antonarakis S. Mutation nomenclature extensions and suggestions to describe complex mutations: A discussion. Hum Mutat 15: 7-12, 2000. 
16. Lane D, Olds R, Conard J, Sas G. Pleiotropic effects of antithrombin strand 1C substitution mutations. J Clin Invest 90: 2422-2433, 1992.

17. Okajima K, Abe H, Maeda S, et al. Antithrombin III Nagasaki (Ser116-Pro): a heterozygous variant with defective heparin binding associated with thrombosis. Blood 81: 1300-1305, 1993.

18. Komamura K, Iwai N, Kokame $K$, et al. The role of a common TNNT2 polymorphism in cardiac hypertrophy. J Hum Genet 49: 129-133, 2004.

19. Strelkov SV, Schumacher J, Burkhard P, Aebi U, Herrmann H. Crystal structure of the human lamin A coil 2B dimer: Implications for the head-to-tail association of nuclear lamins. J Mol Biol 343: 1067-1080, 2004.

20. Bonne G, Mercuri E, Muchir A, et al. Clinical and molecular ge- netic spectrum of autosomal dominant Emery-Dreifuss muscular dystrophy due to mutations of the lamin A/C gene. Ann Neurol 48: 170-180, 2000.

21. Sinagra G, Rakar S, Zecchin M, et al. Nonpredictive value of fibrosis in dilated cardiomyopathy treated with metoprolol. Cardiovasc Path 5: 21-28, 1996.

22. Hayashi M, Matsushita T, Mackman N, et al. Fatal thrombosis of antithrombin-deficient mice is rescued differently in the heart and liver by intercrossing with low tissue factor mice. J Thromb Haemost 4: 177-185, 2006.

23. Mestroni L, Rocco C, Gregori D, et al. Familial dilated cardiomyopathy: Evidence for genetic and phenotypic heterogeneity. J Am Coll Cardiol 34: 181-190, 1999.

\footnotetext{
(C) 2008 The Japanese Society of Internal Medicine http://www.naika.or.jp/imindex.html
} 